\title{
Multistep organic synthesis leading to the formation of triazinothiazoloquinoxalines involving cost effective reagents
}

\author{
Amar SinghYadav *, V.K.Pandey \\ Department of Chemistry, University of Lucknow, Lucknow, India \\ *Corresponding authorE-mail:singh.amar92@gmail.com
}

\begin{abstract}
A simple and efficient chemical method has been attempted for the synthesis of biologically and commercially important 2-aryl [-s-] triazino [1], [3], [5] thiazolo [6], [5-b] quinoxalin -4- thiones in moderate to excellent yields through cyclization reaction of ophenylenediamino using readily available and fewer costly reagents viz. oxalic acid, PCl5thiourea, aromatic aldehydes and ammonium thiocyanate at an ambient temperature.
\end{abstract}

Keywords: $\beta$-Lactamase; Brevity, 2; 3-Dichloroquinoxaline.

\section{Introduction}

At the moment, much emphasis is being laid on the drug design through conjunction, which is known as the systematic formulation of analogues of a prototype agent, in general toward structurally more complex products, which may be viewed as structures embodying, in a general specific way, certain or all the features of the prototype. In this type of drug design, the main principle involved is the 'principle of mixed moieties' i.e. a drug molecule is essentially made up with two or more pharmacophoric moieties embedded into a single molecule. Based on this principle a synthesis of 2-aryl [-s-] triazino [1], [3], [5]thiazolo [6], [5-b] quinoxalin -4- thiones (Scheme 1) has been undertaken. Thus, theazole nucleus constitute a major part of all available penicillin related antibiotics, which interfare with a critical series of proteases involved in bacterial cell wall construction [1].

It is though the production of one of a series of enzyme collectively known as $\beta$-lactamases [2].Within the past decades; several pharmaceutical companies have developed drugs which act as $\beta$ lactamase inhibitors. The known mechanism of inhibition usually involves a nonreversible acylation of serine hydroxyl at an active site [3]. In addition a number of quinoxalinederivatives have been shown to passes a wide variety of pharmacological properties like antibacterial [4], antifungal [5], antituberculosis [6], antitumor [7] antiviral [8] and antiflammatiroy [9]. Further s-triazines have been very well established as diuretics [10] and antiviral agents [11-14] Several s-triazine compounds have been thoroughly examined in neoplastic diseases with promising results but despite the considerable efforts denoted to these compounds, they have not proven to have any clinical application [15], [16]

\section{Results and discussion}

Our initial effort was to carry out a cyclization reaction leading to the formation of quinazolines-2,3 $(1 \mathrm{H}, 4 \mathrm{H})$ dione (1a) with the interaction of $\mathrm{O}$-phenylenediamine with oxalic acid dehydrate using $4 \mathrm{~N} \mathrm{HCl}$ and $10 \% \mathrm{NaOHsoltion}$. This compound existed in tatomeric form (Quinoxaline - 2,3-diol 1b) which on fusion with $\mathrm{PCl}_{5}$ afforded2,3-dichloroquinoxaline (2). For the construction of thiazolo[5], [4-b] qiompxalin-2-amine (3), the protocol adopted was to allow the reaction of 2 with thiourea in absolute alcohol. This was a cycllization reaction, and the cyclized product (3) on reaction with aromatic aldehydes resulted in $\mathrm{N}$-arylidenethiazolo [5], [4-6] quinoxalin - 2 - amines (4a-c). (Table 1) The target compounds (5a-e) (Table 2) designated as 2-aryl - $s=$ triazino[1], [3], [5] - thiazolo [6], [5 -b] quinoxalin-4-thiones were prepared by refluxing a mixture of 4 with thiocyanate in dioxane solvent. All the synthesized compounds have been characterized by IR, ${ }^{1} \mathrm{H}$ NMR, [13]C NMR mass and element analysis. The spectral and analytical data of new compounds (4a-e and 5a-e) are presented in experimental section. In conclusion, we have attempted the synthesis of target molecules involving three cyclization reaction using readily available and fewer costly reagents viz. oxalic acid, thiourea and ammonium thiocyanate Thus considerable attempts have been made to use minimum catalyst and lessexpensivesolvents in the accomplishment of synthesis i.e. brevity and efficiency consideration have been maintained to a greater extent.

Table 1: Synthesis of N-Arylidenethizlolo [5], [4-B] Quinoxalin - 2 Amines

\begin{tabular}{llll}
\hline Entry & Compound & $\mathrm{R}$ & Yield \\
\hline 1 & $4 \mathrm{a}$ & $\mathrm{C}_{6} \mathrm{H}_{5}$ & 75 \\
2 & $4 \mathrm{~b}$ & $4-\mathrm{OH} \mathrm{C}_{6} \mathrm{H}_{4}$ & 70 \\
3 & $4 \mathrm{c}$ & $4-\mathrm{OCH}_{3} \mathrm{C}_{6} \mathrm{H}_{4}$ & 70 \\
4 & $4 \mathrm{~d}$ & $2-\mathrm{OH} \mathrm{C}_{6} \mathrm{H}_{4}$ & 65 \\
5 & $4 \mathrm{e}$ & $4-\mathrm{OH} 3-\mathrm{OCH}_{3} \mathrm{C}_{6} \mathrm{H}_{3}$ & 60 \\
\hline
\end{tabular}

Table 2: Synthesis of 2-Aryl [-S-] Triazino [1], [3], [5] Thiazolo [6], [5-B] Quinoxalin -4- Thiones

\begin{tabular}{llll}
\hline Entry & Compound & $\mathrm{R}$ & Yield \\
\hline 1 & $5 \mathrm{a}$ & $\mathrm{C}_{6} \mathrm{H}_{5}$ & 70 \\
2 & $5 \mathrm{~b}$ & $4-\mathrm{OH} \mathrm{C}_{6} \mathrm{H}_{4}$ & 72 \\
3 & $5 \mathrm{c}$ & $4-\mathrm{OCH}_{3} \mathrm{C}_{6} \mathrm{H}_{4}$ & 82 \\
4 & $5 \mathrm{~d}$ & $2-\mathrm{OH} \mathrm{C} \mathrm{H}_{4}$ & 70 \\
5 & $5 \mathrm{e}$ & $4-\mathrm{OH}, 3-\mathrm{OCH}_{3} \mathrm{C}_{6} \mathrm{H}_{3}$ & 65 \\
\hline
\end{tabular}




\section{Experimental}

All the chemicals were used as received without any further purification. Melting points were determined in open capillaries using a Toshniual melting point apparatus and are uncorrected IR spectra in $\mathrm{KBr}$ were recorded on a Perkin-Elmer 157 Spectrophotometer $\left(v_{\max }\right.$ in $\left.\mathrm{cm}^{-1}\right),{ }^{13} \mathrm{C}$ NMR and ${ }^{1} \mathrm{H}$ NMR spectra in $\mathrm{CDCl}_{3}$ on a varian. $\mathrm{A}_{60} \mathrm{D}$ instrument $(300 \mathrm{MHz})$ using TMS as internal Standard (Chemical shift in $\delta p p m)$. The FAB mass spectra was recorded on JEDL Sx 102/DA-600 mass spectrometer/Data system using Argon/Xenon $(6 \mathrm{KV}, 10 \mathrm{~mA})$ as the FAB gas. The accelerating voltage was $10 \mathrm{KV}$, and the spectra were recorded at room temperature. m-Nitrobenzyl alcohol (NBA) was used as the matrix unless specified otherwise. The matrix peaks may have appeared at $\mathrm{m} / \mathrm{z} 136,137,154,289,307$ in the absence of any metal ions. Elemental analysis were performed on a Perkin-Elmer $240 \mathrm{CHN}$ elemental analyzer. Homogeneities of the compounds were checked using silica gel coated TLC plates and Iodine vapour as visualizing agent.

(1). Quinoxaline-2,3-(1H, 4H) - dione (1)-

A mixture of o-phenylenediamine $(100 \mathrm{mmol})$, oxalic acid dihydrate $(120 \mathrm{mmol})$ and $\mathrm{HCl}(4 \mathrm{~N}, 200 \mathrm{ml})$ was heated to boiling for half an hour. The reaction mixture was cooled to room temperature. A solid separated out which was filtered off and dissolved in $10 \% \mathrm{NaOH}$ solution. It was left overnight after placing animal charcoal to it. It was filtered off and the filtrate was acidified with concentrated $\mathrm{HCl}$. The precipitate which was obtained in this manner was dried at $100^{\circ} \mathrm{C}$ and recrystallized from rectified Spirit m.p. $>300^{\circ} \mathrm{C}\left[>300^{\circ} \mathrm{C}\right][17]$ yield $91 \%$.

(2).2,3-dichloroquinoxaline (2)-

Quinoxalines-2,3-[1H,4H)-dione $(50 \mathrm{mmol})$ was fused with phosporouspentachloride $\left(\mathrm{PCl}_{5}\right)(100 \mathrm{mmol})$ for $2 \mathrm{~h}$. Subsequently, the molten mass was cooled to room temperature. Cold water $(100 \mathrm{ml})$ was added and gently heated. The solid materia was filtered off and dried in air. It was recrystalllized from ethenol as white crystalline mass m.p. $>300^{\circ} \mathrm{C}\left[>300^{\circ} \mathrm{C}\right][18]$ yield $80 \%$

(3).Thiazolo [5], [4-b] quinoxalin - 2 - amine (3) -

A mixture of 2,3 - dichloroquinoxaline $(20 \mathrm{mmol})$ and thiourea $(20 \mathrm{mmol})$ in absolute ethanol $(100 \mathrm{ml})$ was heated under reflex for $2 \mathrm{hr}$. The reaction mixture on cooling and neutralization with anhydrous potassium carbonate solution yielded the free base which on crystallization from dimethyl formamide (DMF) furnished light yellow crystalline mass m.p. $>300^{\circ} \mathrm{C}\left[>300^{\circ} \mathrm{C}\right][19]$ yield $65 \%$

(4).General procedure for the synthesis of $\mathrm{N}$-arylidenethiazolo [5], [4-b] quinoxalin-2-amines (4a-e). -

Thiazolo[5], [4-b] quinoxalin - 2 - amines $(10 \mathrm{mmol})$ and an aromatic aldehydes $(10 \mathrm{mmol})$ in DMSO $(100 \mathrm{ml})$ were heated under reflux for $2 \mathrm{~h}$. Subsequently, Solvent was removed by distillation and the pasty mass was triturated with petroleum ether. It was washed with a solution of sodium carbonate (10\%), Solidification occurred and the solid thus separated out was washed with cold water and dried under vacuum. It was recrystallized from diluted ethanol.

(5).N-Benzylidenethiazolo [5], [4-b] quinoxalin-2-amine (4a) Yellow coloured solid; m.p. $160^{\circ} \mathrm{C}$; $v_{\max }\left(\right.$ film, $\left.\mathrm{cm}^{-1}\right)$ : 3050,1645 , $1575,1490,1449,1240,1035 ;{ }^{1} \mathrm{Hnmr}\left(\mathrm{CDCl}_{3}\right) ; \delta$ 6.70-7.40 (m, $9 \mathrm{H}, \mathrm{Ar}-\mathrm{H}), 8.50(\mathrm{~S}, 1 \mathrm{H}, \mathrm{N}=\mathrm{C}-\mathrm{H}),[13] \mathrm{Cnmr}\left(\mathrm{CDCl}_{3}\right): \delta 155.5$, $141.5,130.9,128.7,128.1,127.4,123.3,117.5 ; \mathrm{MS} \mathrm{m} / \mathrm{z} 290$ $\left(\mathrm{M}^{+}\right)$; Anal. calcd for $\mathrm{C}_{16} \mathrm{H}_{10} \mathrm{~N}_{4} \mathrm{~S}: \mathrm{C}, 66.20 ; \mathrm{H}, 3.44 ; \mathrm{N}, 19.31$. Found: C, 66.18; H, 3.46; N, 19.40

(6).N-4 Hydroxybenzylidenethiazolo [5], [4 - b] quinoxalin - 2 amine (4b) -

Brown coloured solid; m.p, $231^{\circ} \mathrm{C} ; v_{\max }\left(\right.$ film, $\left.\mathrm{cm}^{-1}\right)$ : 3605, 3045, $1642,1570,1495,1445,1235,1195,1032,710 ;{ }^{1} \mathrm{Hnmr}\left(\mathrm{CDCl}_{3}\right) ; \delta$ 6.85-7.76 (m, 8H, Ar -H), $8.54(\mathrm{~S}, 1 \mathrm{H}, \mathrm{N}=\mathrm{C}-\mathrm{H}), 4.65(\mathrm{~S}, 1 \mathrm{H}, \mathrm{Ar}-$ $\mathrm{OH}) ;{ }^{13} \mathrm{Cnmr}\left(\mathrm{CDCl}_{3}\right): \delta 153.6,141.2,137.5,131.4,125.7,121.3$, 117.5, 115.2; MS m/z $306\left(\mathrm{M}^{+}\right)$; Anal. calcd for $\mathrm{C}_{16} \mathrm{H}_{10} \mathrm{~N}_{4} \mathrm{OS}$ : C, $62.74 ; \mathrm{H}, 3.26 ; \mathrm{N}, 18.30$. Found: C, 62.82; H, 3.14; N, 18.42 .
(7).N-4 Methoxybenzylidenethiazolo [5], [4 - b] quinoxalin - 2 amine (4c) -

Dark brown solid; m.p, $281-282^{\circ} \mathrm{C} ; v_{\max }\left(\right.$ film, $\left.\mathrm{cm}^{-1}\right)$ : 3046, 1645 , 1572, 1492, 1450, 1235, 1125, 1032; ${ }^{1} \mathrm{Hnmr}\left(\mathrm{CDCl}_{3}\right) ; \delta$ 6.65-7.67 $(\mathrm{m}, 8 \mathrm{H}, \mathrm{Ar}-\mathrm{H}), 8.61(\mathrm{~S}, 1 \mathrm{H}, \mathrm{N}=\mathrm{C}-\mathrm{H}), 3.75\left(\mathrm{~S}, 1 \mathrm{H}, \mathrm{Ar}-\mathrm{OCH}_{3}\right)$; ${ }^{13} \mathrm{Cnmr}\left(\mathrm{CDCl}_{3}\right): \delta 154.7,140.8,136.5,133.2,131.5,127.4,125.6$, 117.2, 114.4, 39.1; MS m/z $320\left(\mathrm{M}^{+}\right)$; Anal. calcd for $\mathrm{C}_{17} \mathrm{H}_{12} \mathrm{~N}_{4} \mathrm{OS}$ C, 63.75; H, 3.75; N, 17.50. Found: C, 63.54; H, 3.65; N, 17.62. (8).N-2 Hydroxybenzylidenethiazolo [5,4-b] quinoxalin - 2 amine (4d) -

Brown coloured solid; m.p. $>300^{\circ} \mathrm{C}$; $v_{\max }\left(\right.$ film, $\left.\mathrm{cm}^{-1}\right): 3608,3055$, $1645,1572,1492,1450,1238,1190,1036,720 ;{ }^{1} \mathrm{Hnmr}\left(\mathrm{CDCl}_{3}\right) ; \delta$ 6.80-7.72 (m, 8H, Ar -H), $8.50(\mathrm{~S}, 1 \mathrm{H}, \mathrm{N}=\mathrm{C}-\mathrm{H}), 4.62(\mathrm{~S}, 1 \mathrm{H}, \mathrm{Ar}-$ $\mathrm{OH}) ;{ }^{13} \mathrm{Cnmr}\left(\mathrm{CDCl}_{3}\right): \delta 168.5,145.2,141.5,139.5,137.1,131.7$, 127.5, 125.4, 121.2, 119.0, 115.6, 112.5; MS m/z $306\left(\mathrm{M}^{+}\right)$; Anal. calcd for $\mathrm{C}_{16} \mathrm{H}_{10} \mathrm{~N}_{4} \mathrm{OS}$ : C, 62.74; H, 3.26; N, 18.30. Found: C, $62.70 ; \mathrm{H}, 3.14 ; \mathrm{N}, 18.42$.

(9). N-4- Hydroxy-3-methoxy benzylidenethiazolo[5,4-b] quinoxalin (4e) -

Brown coloured solid; m.p. $>300^{\circ} \mathrm{C}$; vmax (film, cm-1): 3605 , 3045, 1642, 1570, 1496, 1440, 1230, 1195, 1120, 1032, 760; $1 \mathrm{Hnmr}(\mathrm{CDCl} 3) ; \delta$ 6.50-7.62 (m, 7H, $\mathrm{Ar}-\mathrm{H}), 8.48(\mathrm{~S}, 1 \mathrm{H}, \mathrm{N}=\mathrm{C}-$ $\mathrm{H}), 4.60 \quad(\mathrm{~S}, \quad 1 \mathrm{H}, \quad \mathrm{Ar}-\mathrm{OH}) 3.70 \quad(\mathrm{~S}, \quad 3 \mathrm{H}, \quad \mathrm{Ar}-\mathrm{OCH} 3)$; 13Cnmr(CDCl3): $\delta$ 166.4, 145.5, 141.4, 139.1, 135.4, 131.7, 129.2, 125.4, 121.4, 117.6,116.2, 115.2, 111.9; MS m/z 336 (M+); Anal. calcd for C17H12N4O2S: C, 60.71; H, 3.57; N, 16.66. Found: C, $60.62 ; \mathrm{H}, 3.68 ; \mathrm{N}, 16.68$.

(10). General procedure for the Synthesis of 2-aryl-s-triazino[1], [3], [5]-thiazolo [6], [5-b] quinoxalin - 4 - thiones (5a-e) -

$\mathrm{N}$-arylidenethiazolo[5], [4-b] quinoxalin - 2 - amine 4 (8mmol) and ammonium thiocyanate $(5 \mathrm{mmol})$ were dissolved in dioxane $(15 \mathrm{ml})$ by warming slowly and stirring. The resultant solution arestirrerfor half an hour at room temperature and subsequently heated under reflux for $5 \mathrm{~h}$ under anhydrous conditions solvent uses removed by distillation and the solid thus obtained was washed successively with cold water. It was dried in vacuo and recrystallized from ethanol.

(11).2-Phenyl-S-triazino [1], [3], [5] - thiazolo [6,5-b] quinoxalin 4 - thione (5a) -

Pale yellow solid; m.p. $>300^{\circ} \mathrm{C} ; v_{\max }\left(\right.$ film, $\left.\mathrm{cm}^{-1}\right)$ : 3320, 3025, $2565,1625,1125, ;{ }^{1} \mathrm{Hnmr}\left(\mathrm{CDCl}_{3}\right) ; \delta$ 7.15-7.91 (m, 9H, $\left.\mathrm{Ar}-\mathrm{H}\right)$, 5.45 (brs, $1 \mathrm{H}, \quad \mathrm{S}=\mathrm{C}-\mathrm{NH}), \quad 5.95 \quad(\mathrm{~S}, \quad 1 \mathrm{H}, \quad \mathrm{S}-\mathrm{H})$; ${ }^{13} \mathrm{Cnmr}\left(\mathrm{CDCl}_{3}\right): \delta 175.5,164.6,147.6,143.5,139.7,131.6,127.4$, 121.1,117.6, 115.2; MS m/z $349\left(\mathrm{M}^{+}\right)$; Anal. calcd for $\mathrm{C}_{17} \mathrm{H}_{11} \mathrm{~N}_{5} \mathrm{~S}_{2}$ : C, 58.45; H, 3.15; N, 20.05. Found: C, 58.35; H, 3.16; N, 20.08.

(12). (4-hydroxyphenyl)-S-triazino[1], [3], [5]-thiazolo[6], [5-b] quinoxalin-4-thione (5b)-

Dark Brown solid; m.p. $>250-251^{\circ} \mathrm{C} ; v_{\max }\left(\right.$ film, $\left.\mathrm{cm}^{-1}\right): 3610,3325$, 3050, 2562, 1635, 1350, $1165, ;{ }^{1} \mathrm{Hnmr}\left(\mathrm{CDCl}_{3}\right) ; \delta$ 7.25-7.95 (m, $8 \mathrm{H}, \mathrm{Ar}-\mathrm{H}), 5.05$ (S, 1H, Ar-OH), 5.40 (brs, $1 \mathrm{H}, \mathrm{S}=\mathrm{C}-\mathrm{NH}), 5.95(\mathrm{~S}$, $1 \mathrm{H}, \mathrm{S}-\mathrm{H}) ;{ }^{13} \mathrm{Cnmr}\left(\mathrm{CDCl}_{3}\right): \delta 164.6,157.6,149.6,143.5,141.4$, 139.7, 134.6, 129.6, 127.3, 124.2, 118.4, 115.5, 111.1; MS m/z $365\left(\mathrm{M}^{+}\right)$; Anal. calcd for $\mathrm{C}_{17} \mathrm{H}_{11} \mathrm{~N}_{5} \mathrm{OS}_{2}$ : C, 55.89; H, 3.01; N, 20.05. Found: C, 55.90; H, 3.03; N, 19.20.

(13).2-(4-methoxyphenyl)-S-triazino [1], [3], [5]-thiazolo[6], [5b]quinoxalin-4-thione $(5 \mathrm{c})$ -

Brown solid; m.p. $265^{\circ} \mathrm{C}$; $v_{\max }\left(\right.$ film, $\left.\mathrm{cm}^{-1}\right): 3365,3050,2560,1630$, 1128, 1125; ${ }^{1} \mathrm{Hnmr}\left(\mathrm{CDCl}_{3}\right) ; \delta$ 6.95-7.67 (m, 8H, $\left.\mathrm{Ar}-\mathrm{H}\right), 3.75(\mathrm{~S}$, $\left.3 \mathrm{H}, \mathrm{Ar}-\mathrm{OCH}_{3}\right), 5.35$ (brs, $\left.1 \mathrm{H}, \mathrm{S}=\mathrm{C}-\mathrm{NH}\right), 5.87(\mathrm{~S}, 1 \mathrm{H}, \mathrm{S}-\mathrm{H})$; ${ }^{13} \mathrm{Cnmr}\left(\mathrm{CDCl}_{3}\right): \delta 172.4,165.4,147.6,144.5,142.4,139.1,131.6$, 129.7, 127.3, 121.4, 117.1, 41.2; MS m/z $384\left(\mathrm{M}^{+}\right)$; Anal. calcd for $\mathrm{C}_{18} \mathrm{H}_{13} \mathrm{~N}_{5} \mathrm{OS}_{2}$ : C, $56.25 ; \mathrm{H}, 3.38 ; \mathrm{N}, 18.22$, Found: C, 56.10; H, $3.28 ; \mathrm{N}, 18.26$.

(14).2-(2-hydroxyphenyl)-S-triazino[1], [3], [5]-thiazolo[6], [5b]quinoxalin-4-thione(5d) -

Brown solid; m.p.245-246 ${ }^{\circ} \mathrm{C}$; $v_{\max }\left(\right.$ film, $\left.\mathrm{cm}^{-1}\right): 3610,3325,3045$, 2570, 1645, 1120; ${ }^{1} \mathrm{Hnmr}\left(\mathrm{CDCl}_{3}\right) ; \delta$ 6.720-7.95 (m, 8H, Ar -H), 4.95 (S, 1H, Ar-OH), 5.45 (brs, 1H, S=C-NH), 5.77 (S, 1H, S- H); ${ }^{13} \mathrm{Cnmr}\left(\mathrm{CDCl}_{3}\right): \delta 167.5,143.6,141.4,135.5,131.5,128.6,121.2$, 
116.8, 111.5; MS m/z $365\left(\mathrm{M}^{+}\right)$; Anal. calcd for $\mathrm{C}_{17} \mathrm{H}_{11} \mathrm{~N}_{5} \mathrm{OS}_{2}: \mathrm{C}$, 55.89; H, 3.01; N, 19.17, Found: C, 55.70; H, 2.99; N, 19.15 .

(15).2-(4-hydroxy-3-methoxyphenyl)-S-triazino[1], [3], [5]thiazolo[6], [5-b]quinoxalin-4-thione(5e) -

Brown solid; m.p. $>300^{\circ} \mathrm{C}$; $v_{\max }\left(\right.$ film, $\left.\mathrm{cm}^{-1}\right): 3615,3320,3055$, $3025,2575,1647,1125 ;{ }^{1} \mathrm{Hnmr}\left(\mathrm{CDCl}_{3}\right) ; \delta$ 6.90-7.85 (m, 7H, $\mathrm{Ar}-$ $\mathrm{H}), 4.95(\mathrm{~S}, 1 \mathrm{H}, \mathrm{Ar}-\mathrm{OH}), 3.65\left(\mathrm{~S}, 3 \mathrm{H}, \mathrm{Ar}-\mathrm{OCH}_{3}\right), 5.35$ (brs, $1 \mathrm{H}$, $\mathrm{S}=\mathrm{C}-\mathrm{NH}), 6.10(\mathrm{~S}, 1 \mathrm{H}, \mathrm{S}-\mathrm{H}) ;{ }^{13} \mathrm{Cnmr}\left(\mathrm{CDCl}_{3}\right): \delta 165.6,145.5$, 141.4, 139.6, 131.5, 129.8,127.1, 120.2, 117.6, 115.7, 111.5,; MS $\mathrm{m} / \mathrm{z} 395\left(\mathrm{M}^{+}\right)$; Anal. calcd for $\mathrm{C}_{17} \mathrm{H}_{13} \mathrm{~N}_{5} \mathrm{O}_{2} \mathrm{~S}_{2}$ : C, 54.68; H, 3.29; N, 17.72, Found: C, 55.2; H, 3.18; N, 17.70 .

\section{Conclusion}

In conclusion, we have attempted the synthesis of target molecules involving three cyclization reaction using readily available and fewer costly reagents viz. oxalic acid, thiourea and ammonium thiocyanate Thus considerable attempts have been made to use minimum catalyst and less-expensivesolvents in the accomplishment of synthesisi.e. brevity and efficiency consideration have been maintained to a greater extent. An effective drug design from a biologically active prototype, whether approached through disjunction or conjunction or both, normally aims at modifying collectively all the moiety attributes that are essentialcapacities of a drug eliminated to a great extent which otherwise would have reduced its specificity of action, or interference with the primary type of action sought

\section{Acknowledgements}

The authors are thankful to the Head, Department of Chemistry, University of Lucknow for providing necessary laboratory facilities. Thanks are also due to The Director, Central Drug Research Institute (CDRI), Lucknow for providing elemental and spectral data. Author (Dr.amar singh yadav) are thankful to council of Science and Technology Uttar Pradesh, for Financial Support to authors under a young scientist scheme.

\section{References}

[1] Buynak, J.D; Borate, H.B.; Lamb, G.W.;Khasnis, D.D.; Husting, C., Isom, H.; Siriwardane, U. J. Org Chem 1993, 58, 13251335.https://doi.org/10.1021/jo00058a008

[2] (a) Bush, K. Antimicrob. Agents Chemother 1989, 33, 264. (b) Bush, K. Antimicrob. Agents Chemother 1989, 33, 271.https://doi.org/10.1128/AAC.33.3.264.

[3] (a) Knowles, J.R. AccChem Res 1985, 18, 97. (b) Knowles, J.R AccChem Res 1983, 6, 90.https://doi.org/10.1021/ar00112a001.

[4] Wagle, S; Adhikari, A.V.; Kumari, N.S. Ind J Chem2008, 47B, 439.

[5] Khan, S.A.; Salim, K.; Khan, Z. European J Med Chem 2007, 42, 103.https://doi.org/10.1016/j.ejmech.2006.07.006.

[6] Coulthord, C.E.; Hale, L.J.; Gurd, M.R. Brit J Pharmacol 1954, 10, 394.

[7] Francis, J.; Landquist, J.K.; Levi, A.A.; Silk, J. A.; Thorp, J.M. Biochem J, 1956, 63, 455.https://doi.org/10.1042/bj0630455.

[8] Hennessey, T.D.; Edwards, J.R. Vet Rec 1972, 90, 187.https://doi.org/10.1136/vr.90.7.187.

[9] Kotharkar, S.A.; Shinde, D.B. Biorg Med ChemLett 2006, 16, 6181.https://doi.org/10.1016/j.bmcl.2006.09.040.

[10] Waring, M.J.; Ben Hadda, T.; Kotchevar, A.T.; Ramdani, A.; Touzani, R.; Elkadiri, S.; Hakkou, A.; Bouakka, M.; Ellias, T. Molecules, 2002, 7, 641.https://doi.org/10.3390/70800641.

[11] Grande, F.; Aiello, F.; Grazia, O.D.; Brizzi, A.; Garofaloa, A.; Neamati, N.Biorg.Medchem 2007, 15 288.https://doi.org/10.1016/i.bmc.2006.09.073

[12] Lee, I.S.; Waring, M.J. Biochem J 1978, 173, 129.https://doi.org/10.1042/bj1730129.

[13] Waring, M.J.; Wakelin, L.P.G. Nature 1974, 252, 653.https://doi.org/10.1038/252653a0.

[14] Lee, J.S.; Waring, M.J. Biochem J 1978, 173, 115.https://doi.org/10.1042/bj1730115.
[15] Klcim, J.; Bender, R.; Billhardt, U.; Meichsner, C.; Riess, G.; Rosner, M.; Winkler, I.; Paessens, A. Antimicrobial agents and chemotherapy, 1993, 1659.

[16] Yolanda, S.; Elena, M.; Javier, F.; Angel, M.; Gerain, L.D. Arzneimittel-Forschung 1999, 49(1), 55.

[17] Williamson, H.E.; Shiderman, F.E.; Lescher, D.A. J Pharmacol Exp. Ther 1959, 126, 82 .

[18] Glandyil, J.M.Z.; Hunt, Z.H.; Jack, D.; Haff, R,F.; Boyle, J.J.; Steward, R.C.; Ferlanto, R.J. Nature, 1968, 221, 286.

[19] Boyle, J.J.; Ravpp, W.G.; Stanfield, F.J.; Haff, R.F.; Diek, E.C.; D' Allesio, D; Dick, C.R. Ann N Y AcadSci 1970, 173 477.https://doi.org/10.1111/j.1749-6632.1970.tb53436.x.

[20] Gwaltney, T.M. ProcSocExpBiol Med 1970, 133 1148https://doi.org/10.3181/00379727-133-34642.

[21] Haff, R.F.; Flagg, W.B.; Gallo, J.J.; Hoover, J.R.E.; Miller, J.A.; Pinto, C.A.; Pegano, J.F. OrocSocExpBiol 1972, 141, 475.

[22] Sorm, F.; Vesely, J. Neoplasm, 1964, 11, 123.

[23] Jurovcik, M.; Raska, K.; Sormova, J.; Sorm, P. Collect Czech chem common 1965, 20, 3370.

[24] Mohan, J; Kumar, A. Ind J Het Chem 2003, 12, 283-284.

[25] Mohan. J. IndJHetchem 1998, 32, 953.

[26] Upadhyay, M. in "Synthesis of some fused ring heterocyclic Compounds for studying their biological activity" A Ph.D thesis, Chemistry Department, Lucknow University, Lucknow.P.159 (2005). 
Scheme I

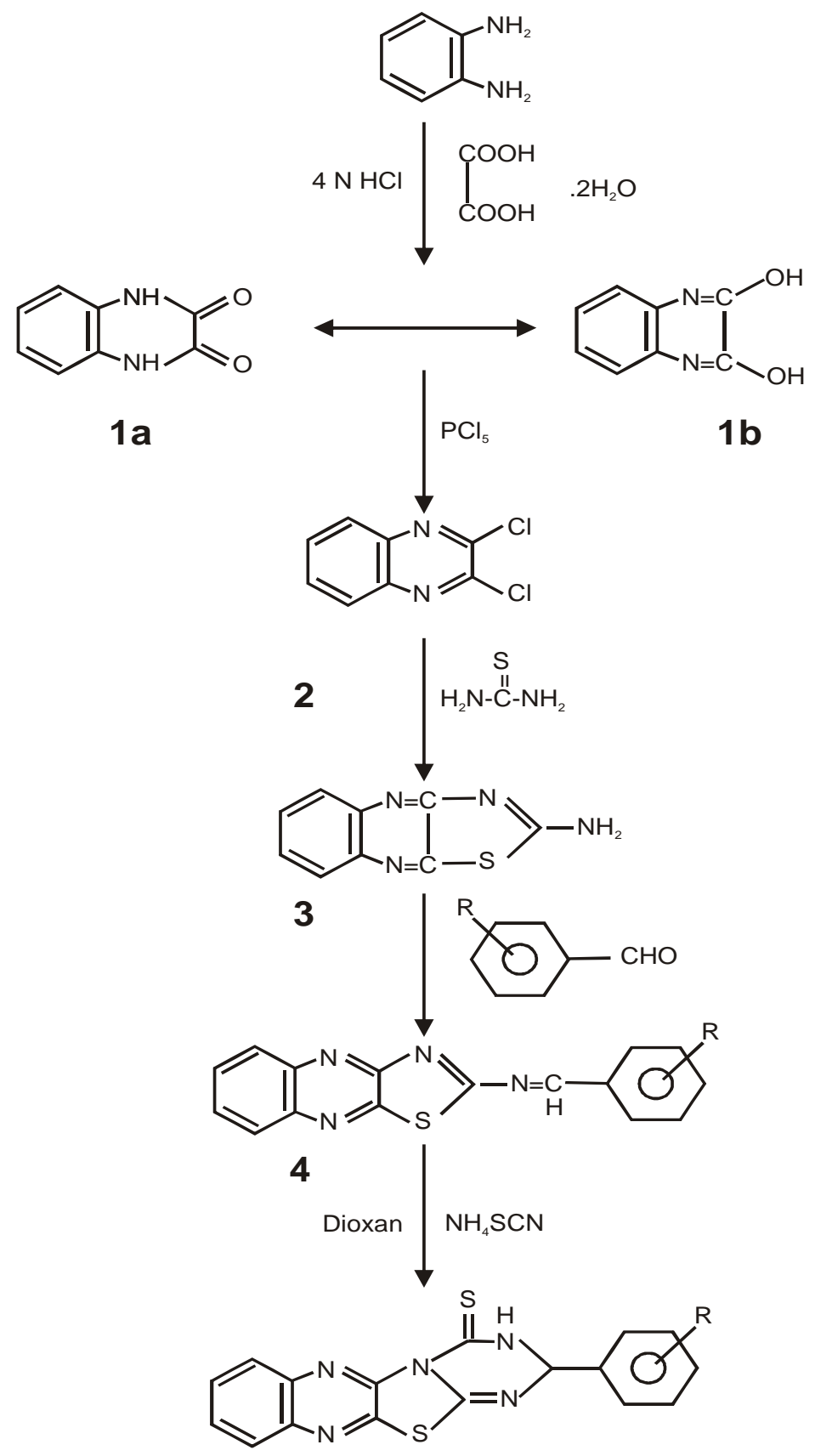

